\title{
Stellar velocity dispersion in NLS1 galaxies
}

\author{
V. Botte ${ }^{1}$, S. Ciroi ${ }^{1}$, F. Di Mille ${ }^{1}$, P. Rafanelli ${ }^{1}$ and A. Romano ${ }^{1}$ \\ ${ }^{1}$ Department of Astronomy, University of Padua, Vicolo dell'Osservatorio, 2, \\ I-35122 Padova, Italy
}

\begin{abstract}
Measuring stellar velocity dispersion $(\sigma)$ in Narrow-Line Seyfert 1 (NLS1) with optical spectra, can be very difficult because of the large and bright Fe II multiplets, which completely suppress the typically used stellar absorption lines. Indeed, $\sigma$ values are to date available only for 2 NLS1s. Here we present new $\sigma$ measurements of three NLS1s observed in the spectral range centered around the Ca II triplet $(\lambda \sim 8600 \AA)$. Moreover, we compare $\sigma$ with the gaseous velocity dispersion finding that the $\sigma=\mathrm{FWHM}([\mathrm{O}$ III $] 5007 \AA) / 2.35$ relation (Nelson \& Whittle 1995) is not satisfied by our galaxies.
\end{abstract}

\section{Introduction}

In the last years a great interest was addressed to the study of the correlation between the mass of nuclear supermassive black-holes $\left(M_{B H}\right)$ and the stellar velocity dispersion $(\sigma)$ of their hosting bulges. On this topic no consensus has been reached until now about Narrow-Line Seyfert 1 (NLS1) galaxies. These active nuclei (AGN) are believed to host BHs with smaller masses than Seyfert 1 (S1) galaxies, accreting at higher rates, close to the Eddington limit. This current paradigm implies that NLS1s would have also less massive bulges than S1s, under the assumption that they follow the $M_{B H}-M_{b u l g e}$ correlation. Wang \& $\mathrm{Lu}(2001)$ found that there is no clear difference in the $M_{B H}-$ $\sigma$ relation between narrow-line, broad-line AGN and non-active nearby galaxies, while opposite results were obtained by Mathur et al. (2001), Grupe \& Mathur (2004) and Botte et al. (2004), who found that NLS1s and S1s span similar ranges of $\sigma$, suggesting that their bulges are in this respect identical. Moreover, all NLS1s of their sample fall below the recently obtained correlations (see e.g. Tremaine et al. 2002), showing $\sigma$ values higher than expected. A possible explanation to this disagreement could be the fact that in NLS1s, $\sigma$ is generally measured in terms of FWHM([O III] $] 5007 \AA)$ (Nelson \& Whittle 1995), given the presence of Fe II multiplets, which hide the commonly used stellar absorption lines. Actually, $\sigma$ values are available only for 2 NLS1s: Mrk 590 ( $\sigma=$ $\left.169 \pm 22 \mathrm{~km} \mathrm{~s}^{-1}\right)$ and NGC $4051\left(\sigma=88 \pm 13 \mathrm{~km} \mathrm{~s}^{-1}\right)$ (Nelson \& Whittle 1995). Moreover Nelson (2000) claimed that the $M_{B H}$ of AGN for which $\sigma$ was derived from the [O III] width is in agreement with the $M_{B H}-\sigma$ relation defined for nearby galaxies. More recently, Boroson (2003) confirmed this result using the spectra of 107 low-redshift radio-quiet QSOs and S1s, extracted from the Sloan Digital Sky Survey.

\section{Aims and First Results}

Since the gaseous component is not always expected to respond to the gravitational potential as stars do, we suggest that the use of the [O III] width as a surrogate for $\sigma$ might not be appropriate for NLS1s. Our aim is to obtain a direct determination of $\sigma$ in NLS1 nuclei by means of the absorption lines of the Ca II triplet $(\sim 8600 \AA)$, in order to verify if their values are on average lower than those measured for S1s (150$300 \mathrm{~km} \mathrm{~s}^{-1}$ ). If this is not the case, it is possible that the $M_{B H}-\sigma$ relation is simply not 

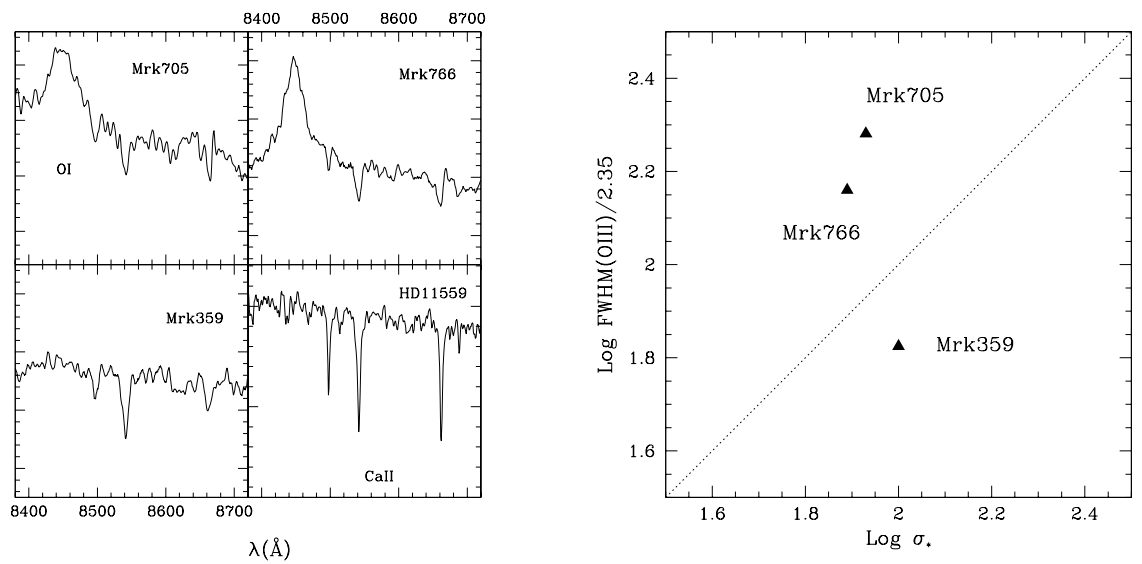

Figure 1. Preliminary results of 3 NLS1s: Mrk 359, Mrk 705, Mrk 766 (left); stellar vs. gas velocity dispersion for the same NLS1s: there is no clear correlation with respect to the 1:1 line (right)

followed by NLS1s, or in other words, that NLS1s have a lower $M_{B H} / M_{\text {bulge }}$ ratio (Botte et al. 2004). At the $1.82 \mathrm{~m}$ telescope of the Padova Astronomical Observatory (Asiago, Italy) we have observed a sample of NLS1s in the 8000-9000 $\AA$ range with relatively high spectral resolution $\left(\sigma \sim 50 \mathrm{~km} \mathrm{~s}^{-1}\right)$. The spectra of three NLS1s: Mrk 359, Mrk 705 and Mrk 766, are shown in Fig. 1 (left). We used the Fourier cross-correlation method for determining the velocity dispersion (Tonry \& Davis 1979). The spectrum of the galaxy and the stellar template are cross-correlated in Fourier space, and the resulting peak is fitted by a smooth symmetric function. The peak position and the width of the function are related to the galaxy redshift and stellar velocity dispersion, respectively.

In order to verify if the FWHM of [O III $] 5007 \AA$ is really a good representation of the bulge velocity dispersion in NLS1s, we plotted $\sigma$ values vs. the [O III] widths (Fig. 1, right). At least two considerations are possible at this stage: firstly, this preliminary analysis shows clearly that the use of Ca II absorption lines is a good choice to measure stellar kinematics in NLS1s and the resolution of our spectra is sufficiently high to produce reliable values. Secondly, even if three points are not statistically significant, two of them are located far from the $\sigma=\mathrm{FWHM}([\mathrm{OIII}] 5007 \AA) / 2.35$ line, and above it, that is the stellar velocity dispersion is overestimated in these two galaxies when the [OIII] width is used.

\section{References}

Boroson, T. A. 2003, ApJ, 585, 647

Botte, V., Ciroi, S., Rafanelli, P., \& Di Mille, F. 2004, AJ, in press

Grupe, D., \& Mathur, S. 2004, (astro-ph/0312390)

Mathur, S., Kuraszkiewicz, J., \& Czerny, B. 2001, New Astronomy, 6, 321

Nelson, C. H. 2000, ApJ, 544, L91

Nelson, C. H., \& Whittle, M. 1995, ApJS, 99, 67

Nelson, C. H., \& Whittle, M. 1996, ApJ, 465, 96

Tonry, J., \& Davis, M. 1979, AJ, 84, 1511

Tremaine, S., et al. 2002, ApJ, 574, 740

Wang, T., \& Lu, Y. 2001, A\&A, 377, 52 